\title{
Intervención ante prejuicios estéticos y condiciones físicas en niños y niñas de 5 años ${ }^{5}$
}

\author{
María Emilia D'Amanzo \\ Licenciada en Psicología \\ Facultad de Humanidades y Ciencias Económicas \\ Pontificia Universidad Católica de Argentina \\ Correo electrónico: emiliadamanzo@gmail.com
}

\author{
Leandro Martín Casari \\ Doctor en Psicología \\ Instituto de Ciencias Humanas, \\ Ambientales y Sociales \\ Consejo Nacional de Investigaciones \\ Científicas y Técnicas \\ Correo electrónico: leandromartincasari@gmail.com
}

Recibido: 15/01/2019

Evaluado: $24 / 05 / 2019$

Aceptado: 18/07/2019

\section{Resumen}

Esta investigación tuvo como propósito indagar la posibilidad de disminuir los prejuicios relacionados con los estereotipos estéticos y condiciones físicas en los niños/as. Para esto, en primer lugar, se desarrolló un instrumento que evalúa si los niños/as poseen prejuicios. A partir de los resultados obtenidos, se implementó un programa de intervención, el cual consta de talleres con actividades lúdicas para enseñarles a los niños y niñas una manera distinta de ver a sus pares, y así evitar discriminaciones a futuro. La muestra estuvo compuesta por 42 niños de ambos sexos, de 5 años de edad. Los resultados mostraron que los niños y niñas poseían prejuicios, los cuales fueron notablemente disminuidos luego de la aplicación de los talleres.

Palabras clave Intervención, prejuicios, niños.

5 Para citar este artículo: D'Amanzo, M. E. y Casari, L.M. (2021). Intervención ante prejuicios estéticos y condiciones físicas en niños de 5 años. Informes Psicológicos, 21(1), 73-86 http://dx.doi.org/10.18566/infpsic.v21n1a05 


\title{
Intervention against aesthetic prejudices and physical conditions in 5-year-old children
}

\begin{abstract}
The purpose of this research was to investigate the possibility of reducing prejudices related to aesthetic stereotypes and physical conditions in children. For this, in the first place, an instrument was developed to assess whether children are prejudiced. Based on the results obtained, an intervention program was implemented, which consists of workshops with recreational activities to teach boys and girls a different way of seeing their peers and thus avoid discrimination in the future. The sample consisted of 42 children of both sexes, 5 years old. The results showed that both boys and girls had prejudices, which were notably diminished after the application of the workshops.
\end{abstract}

Keywords

Intervention, prejudices, children.

\section{Intervenção perante preconceitos estéticos e condições físicas em crianças de 5 anos}

\section{Resumo}

0 objetivo desta pesquisa foi investigar a possibilidade de reduzir preconceitos relacionados a estereótipos estéticos e condições físicas em crianças. Para isso, em primeiro lugar, foi desenvolvido um instrumento que avalia se as crianças possuem preconceitos. Com base nos resultados obtidos, foi implementado um programa de intervenção, que consiste em oficinas com atividades lúdicas para ensinar às crianças uma forma diferente de ver os seus pares, evitando assim, no futuro, discriminações. A amostra foi composta por 42 crianças de ambos os sexos, com 5 anos de idade. 0s resultados mostraram que meninos e meninas apresentavam preconceitos, que foram notadamente diminuídos após a aplicação das oficinas. 


\section{ntroducción}

El término "prejuicio" es un concepto previo al razonamiento que carece de fundamentación. Está basado en ideas preconcebidas, anticuadas y a menudo erróneas. Así pues, el antídoto contra el prejuicio es que las personas que los posean se familiaricen más con lo que temen, para que sus creencias puedan ser corregidas con datos reales (Instituto Nacional contra la Discriminación, la Xenofobia y el Racismo [INADI], 2011; MentalHealth.net, 2010; Solbes, Enesco, \& Escudero, 2008).

Aboud (1988, citado en Guerrero, 2007) planteó la Teoría Socio-Cognitiva, la cual ha sido la que mayor repercusión ha tenido en la investigación evolutiva del prejuicio. Propone un modelo de estadios en los cuales el prejuicio se expresa de forma cualitativamente distinta según la estructura cognitiva del sujeto. El primer estadio abarca la edad de la muestra seleccionada, durante la cual los niños/as establecen similitudes y diferencias entre sí mismos y otros. Logran clasificar el mundo social en categorías rudimentarias y rígidas, basadas en características puramente físicas, atributos externamente observables (color de piel, ropa, cabello, e incluso el habla) más que en cualidades internas.

Los niños, desde muy pequeños, entre los 3 y 6 años, comienzan a comprender y utilizar los prejuicios aprendiendo a comportarse en base a estos (Elizathe, Murawski, \& Rutsztein, 2011; Lobera, Rivas, Montaña, \& Morales, 2008; Pappas, 2012).
Algunas de las primeras técnicas de investigación aplicadas al estudio de las actitudes en la niñez se desarrollaron en los años 30 y 40 en Estados Unidos. La mayoría de los trabajos se han realizado usando una estructura básica de entrevista semiestructurada, en la que el experimentador muestra distintos estímulos al participante y le pregunta sobre sus preferencias, identificación o atribución de adjetivos, a través de un procedimiento que podríamos considerar clínico (Guerrero, 2007; Solbes, 2010).

En su estudio clásico, Horowitz (1936) se propuso determinar el grado en que los niños eran conscientes de diferentes categorías sociales como la etnia, el género o la clase social, y la importancia que atribuían a cada una de ellas. Con este objetivo, enseñaba a los participantes un conjunto de dibujos que representaban a niños que variaban en estos aspectos, pidiéndoles que agruparan a las figuras "que iban juntas" o que "señalaran las diferentes". Los resultados mostraron que la etnia era la categoría dominante para la mayoría de los niños, seguida del género de las figuras (Guerrero, 2007; Solbes, 2010).

Por su parte, Clark y Clark (1947) residieron en estudiar las preferencias, rechazos y las actitudes que los niños pequeños mostraban hacia personas de distintos grupos raciales, así como el reconocimiento de estos grupos y su identificación con alguno de ellos. Para ello entrevistaron a un grupo de niños blancos y negros de 3 a 7 años utilizando el llamado The Clark Doll Test (Prueba de las muñecas de Clark). Emplearon un par de muñecas de distinto color (blanca/negra) y se les preguntaba, por ejemplo, ¿cuál te gusta más/menos?, ¿con cuál te gustaría 
jugar? o sobre los procesos de identificación, ¿a quién te pareces más? Los resultados generales de este trabajo señalaban que los niños negros mostraban una baja autoestima grupal, apareciendo una marcada preferencia por las muñecas de piel blanca y una tendencia generalizada de rechazo hacia las figuras negras. Además, comprobaron que los niños negros tenían dificultades a la hora de identificarse con su grupo racial, seleccionando la figura blanca como aquella a la que más creían parecerse a menudo. En comparación con estos resultados, los niños blancos mostraban desde muy pronto (entre los 3 y 5 años) una orientación positiva hacia las figuras de piel blanca, así como una correcta identificación con su grupo étnico de pertenencia.

Este procedimiento ha sido replicado en múltiples trabajos, realizando importantes variaciones principalmente centradas en la sustitución de las muñecas por otro tipo de estímulos más realistas como fotografías, recortables o muñecos con distintos rasgos raciales más allá del color de la piel; también se han ampliado las preguntas realizadas en relación a las figuras o muñecas, indagando sobre los atributos adscritos a cada uno de los estímulos (estereotipos), o sobre la selección de compañeros para realizar diferentes actividades (Guerrero, 2007; Solbes, 2010).

El trabajo de Richardson, Goodman, Hastorf, y Dornbusch (1961) fue uno de los primeros en analizar las preferencias de los niños hacia distintas variables físicas de las personas. Estos autores presentaron a un grupo de niños de 10-11 años varios dibujos de niños con distintos problemas físicos (un niño con muletas, en silla de ruedas, manco, con sobrepeso o con la cara desfigurada) pidiéndoles que los colocaran en orden de preferencias. Uno de los resultados más llamativos fue que la mayoría de los participantes situó la figura del niño obeso en los últimos lugares de preferencia. Desde entonces, se han diseñado numerosos cuestionarios o entrevistas en las que se pide a los participantes que respondan evaluando o asignando adjetivos a diferentes tipos de estímulos (dibujos, fotografías) que representan a niños y niñas de distinta complexión física (Latner \& Stunkard, 2003, citados en Solbes, Enesco \& Escudero, 2008).

El procedimiento empleado en la mayoría de las investigaciones sobre este tema ha consistido en la presentación de dibujos o fotos de niños con distinta complexión física: normalmente niños de peso medio frente a niños con sobrepeso. Las tareas que se plantean a los participantes son también equivalentes a las utilizadas en el estudio de los prejuicios étnicos: preferencias y rechazos simples (por ejemplo, ¿cuál te gusta más/menos?) - para realizar actividades concretas (¿con cuál te gustaría más/menos jugar?), identificación (¿a cuál te pareces más/te gustaría parecerte/no te gustaría parecerte?), atribución de adjetivos (¿cuál te parece el más listo/tonto?), las preferencias de compañero de juego (¿con cuál te gustaría más/menos jugar?).

Más recientemente, algunos autores han abordado el estudio del prejuicio a la gordura mediante ingeniosos métodos experimentales en los que se manipula el peso de la persona y se mantienen constantes otras características físicas, lo cual permite realizar inferencias de causalidad y evitar la posible influencia de factores externos en la representatividad 
de los estímulos respecto a la categoría que pretenden evocar. Por ejemplo, se han estudiado las actitudes de niños hacia posibles compañeros presentados en un video que difieren únicamente en su complexión física aparente (i.e. en unos casos llevan "trajes de gordo" mientras que, en otros, no), manteniendo iguales las restantes características.

Se han desarrollado otras pruebas como el Preschool Racial Attitude Measure (PRAM) de Williams, Best, Boswell, Mattson, y Graves (1975) o el Multiresponse Racial Attitude Measure (MRA) de Doyle, Beaudet y Aboud (1988), en el cual se explica al participante una pequeña historia en la que aparecen una serie de personajes que poseen diferentes atributos bipolares (por ejemplo, bueno/malo), pidiéndole que seleccione entre una cantidad variable de estímulos (por ejemplo, fotos de niños blancos y negros) quien podría representar cada uno de los papeles. La idea general que subyace a estas pruebas es que los patrones de atribución de adjetivos que realicen los niños (por ejemplo, si tienden a atribuir los adjetivos negativos a los niños negros) revelaran el grado de prejuicio racial que poseen.

Katz y Zalk en 1978 diseñaron una prueba, el Test Proyectivo sobre prejuicios (Projective Prejudice Test), que consistía en la presentación de una serie de situaciones sociales ambiguas mediante diferentes ilustraciones. Las ilustraciones presentaban varias historias en las que aparecían implicados personajes blancos y negros, uno de los cuales estaba realizando una acción que podría ser interpretada como positiva (por ejemplo, ayudar a un compañero que se había caído) o negativa (como empujarle). Los participantes estudiaban las ilustraciones y daban una interpretación a lo que ocurría en cada caso, proyectando (supuestamente) sus actitudes étnicas en las situaciones que se presentaban. La idea que subyace a esta prueba es que el color de la piel de los personajes influiría en algunos niños (los que fueran prejuiciosos), sesgando su interpretación hacia las intenciones negativas en los casos en los que el personaje que realizaba la conducta ambigua fuera negro, y hacia las intenciones positivas cuando el personaje fuera blanco. Los resultados de este trabajo original confirmaron que la mayoría de los participantes interpretaba de forma más negativa las situaciones ambiguas cuando la historia que observaban tenía como protagonista un niño negro (Guerrero, 2007; Solbes, 2010).

El estudio de Cramer, Steinwert y College (1998) consistió en la aplicación de una entrevista semiestructurada en la que se planteaban distintos tipos de tareas a los participantes, niños de 3 a 5 años de edad. Sus resultados mostraron que, ya a los 3 años, los participantes solían atribuir a la figura del niño con sobrepeso atributos negativos, lo seleccionaban como aquella figura a la que no querían parecerse y lo rechazaban como posible compañero de juego. Sin embargo, cuando se les pedía que justificaran sus elecciones, la mayoría no citaban el exceso de peso como el factor determinante de sus preferencias, cosa que sí hacían los niños de 4 años (Solbes, 2010).

Abrams (2010) afirma que, debido a la naturaleza cambiante de los prejuicios en la infancia, los científicos del desarrollo emplean una variedad de métodos para estudiar los prejuicios de los niños. Tres de ellos son: las preferencias explícitas de los niños para los miembros de sus 
propios grupos frente a otros, a veces reflejadas en juicios sesgados sobre las características de los miembros del grupo; los sesgos implícitos, es decir, los que los niños no pueden controlar y no son necesariamente conscientes de ellos, los cuales surgen temprano en la infancia; y la exclusión o rechazo de los compañeros individuales en contextos intergrupales, examinando cómo los niños toman decisiones acerca de a quién incluir y excluir de sus relaciones sociales en los contextos cotidianos intergrupales.

El estudio más relevante, relacionado con lo mencionado anteriormente, fue realizado por Solbes (2010) y tuvo como objetivo general, estudiar las actitudes hacia la complexión corporal ligada al peso en un grupo de 120 niños y niñas escolarizados. Participaron en el estudio 40 niños de cada uno de los tres cursos seleccionados para esta investigación $\left(1^{\circ}, 3^{\circ}\right.$ y $5^{\circ}$ de Educación Primaria), siendo el número de niños y niñas el mismo en cada grupo de edad (20 en cada caso). Los resultados mostraron que los alumnos poseían claros sesgos negativos hacia los niños con sobrepeso (a nivel explícito e implícito). La mayoría de los niños mostraba una marcada preferencia por pares de peso medio, a los que elegía mayoritariamente como compañeros para realizar distintas actividades y les atribuía gran cantidad de atributos positivos. Por su parte, los niños con sobrepeso fueron mayoritariamente rechazados y recibieron la mayor parte de los adjetivos negativos propuestos.

Para asegurar que las intervenciones sean eficaces y reducir los prejuicios en la infancia, es esencial que se basen en teoría y hallazgos psicológicos, que se realicen de manera temprana, con frecuencia y que sean evaluadas sistemáticamente.
Se propuso como objetivo general indagar la posibilidad de poder disminuir los prejuicios relacionados con los estereotipos estéticos y condiciones físicas en los niños/as, para evitar discriminaciones a futuro; se establecieron como objetivos específicos: 1) indagar, a través del estudio de las variables peso, altura, uso de anteojos y color de piel, los posibles prejuicios que pueden tener los niños/as; y 2) comparar, a través de la realización de talleres, si los prejuicios estéticos cambian con respecto a su visión anterior.

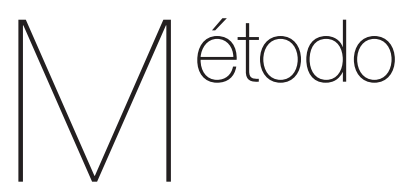

La presente investigación se abordó desde un diseño cuasi experimental (Hernández-Sampieri, Fernández-Collado \& Baptista-Lucio, 2014). Debido a que existe una intervención del investigador sobre la variable dependiente (prejuicios estéticos en niños), los grupos denominados control y experimental no pudieron ser asignados al azar, sino que se escogió la división con mayor cantidad de prejuicios como grupo experimental, y a la otra división como grupo control.

\section{Participantes}

Se trabajó con una muestra de niños y niñas de 5 años que asisten a un jardín de infantes de la ciudad de Godoy Cruz, Mendoza, Argentina. En total participaron 42 niños: 21 (17 niñas y 4 niños) dentro del grupo experimental, con 
el cual se realizaron las intervenciones, y 21 (13 niñas y 8 niños) dentro del grupo control.

\section{Instrumento}

El instrumento que se utilizó es una escala confeccionada Ad Hoc, denominada "Escala de evaluación de prejuicios en la infancia". La misma es utilizada como pre-test y post-test; consta de una serie de 8 tarjetas con imágenes ilustradas de 2 niños o 2 niñas (dependiendo respectivamente del sexo correspondiente del alumno a responder) con distintos patrones de belleza, relacionados con las variables a investigar (peso, color de piel, altura y uso de anteojos). Al observar las tarjetas, las cuales tenían dos personajes exactamente iguales, salvo por la diferencia de las variables mencionadas previamente, los estudiantes debían mencionar con cuál de las dos figuras ilustradas se sentían más a gusto respondiendo ciertas preguntas: ¿con quién te gustaría jugar?, ¿a quién invitarías a tu casa?, ¿cuál de ellos es más bueno? De esta manera, se pueden identificar las nociones de belleza y estética que podrían estar presentes en los alumnos.

\section{Procedimiento}

Como primera aproximación a la investigación, se hizo un estudio cualitativo, relevando en un total de 10 maestras de nivel inicial pertenecientes a escuelas públicas de Godoy Cruz (Argentina) los prejuicios frecuentes en edad pre-escolar. Luego de esto, se armó la escala Ad Hoc con imágenes ilustradas relativas a los prejuicios a evaluar, donde las figuras dibujadas eran diferentes entre sí.
Una vez realizado el instrumento se tuvo un primer contacto con la institución. Se procedió a enviar un consentimiento informado para obtener la autorización de trabajar con los niños/as.

El proceso comenzó a partir de la realización de una prueba piloto, para así poder verificar si el instrumento era lo suficientemente confiable para utilizarlo en la investigación final. Ésta se realizó en una sala de 22 alumnos, 11 niños y 11 niñas, a través del método test-retest, realizando el retest, 15 días posteriores a la toma inicial. Al finalizar esta prueba se pasó a analizar los resultados y el motivo de la elección en los niños/as. También se observó si había o no coincidencia entre la elección del pre y la del post.

Luego se analizó la validez de contenido de la escala utilizada a través del criterio interjueces, enviando el instrumento a profesionales de la salud y educación.

Tras la realización del estudio preliminar y a partir de las correcciones de la escala realizadas en los formatos de jueces expertos, se modificaron ligeramente algunos detalles del instrumento, principalmente aspectos relativos a las figuras de los dibujos diseñados en las tarjetas utilizadas, pasando de tener dibujos diferentes, a ser exactamente iguales con una única variación: la del prejuicio a querer evaluar.

Una vez modificado el instrumento, y nuevamente, luego de enviar a los padres del alumnado el consentimiento informado, se procedió a realizar la toma final del mismo; esto se hizo en dos grupos, ambos mixtos. Finalizada la primera evaluación en los dos grupos, se pasó a elegir cuál sería el grupo experimental y cuál el 
grupo control, teniendo en cuenta como dato de mayor importancia para la elección del mismo, el grupo que poseía mayor cantidad de prejuicios.

El trabajo con el grupo experimental consistió en una serie de 3 talleres. Cada taller estaba compuesto por el inicio, desarrollo (en el cual se realizaban dos actividades lúdicas y recreativas, una por prejuicio a trabajar) y cierre del mismo. Se realizaron dos en el transcurso de una semana y el taller de cierre en la semana siguiente, con una duración de 45 minutos reloj cada día y el último taller de 30 minutos. Los mismos se llevaron a cabo, con el objetivo de poder flexibilizar a los niños sobre afirmaciones y creencias acerca de estereotipos y prejuicios en cuanto a condiciones físicas; y además logren comprender que, a pesar de que sus compañeros cambien a nivel físico, continúan siendo las mismas personas.

El primer taller comenzó con la presentación de cada uno de los integrantes del grupo, luego se comentó y reflexionó sobre la definición básica de discriminación y prejuicio. Una vez comprendido esto, se trabajó con un juego referido al prejuicio del uso de anteojos, para el cual se realizaron anteojos de material goma eva, uno con un modelo distinto para cada niño, los cuales, al finalizar la dinámica, podían llevárselos. El juego consistió en que, previamente escondidos los anteojos por distintos sectores de la escuela, los niños debían buscarlos $y$, una vez encontrados, volver a la sala a ponérselos; cuando cada niño/a se había colocado el suyo debieron mirarse entre todos y reconocerse con los lentes puestos. Luego se les leyeron frases, tales como: "Los niños que usan anteojos no pueden correr", "Los niños que no usan anteojos son lindos", a las que los alumnos debían responder si la afirmación es verdadera o falsa, para lo que se habían llevado emoticones dibujados de un dedo pulgar hacia arriba y un dedo pulgar hacia abajo; además, debían responder cómo se sentirían si esa afirmación se la dijeran a uno, bien o mal (utilizando emoticones de una cara feliz y una cara triste). Posteriormente, se reflexionó sobre la necesidad de algunas personas de utilizar anteojos recetados y no por eso ser feos o no poder jugar a ciertas actividades, y se les mostró también que, con o sin lentes, todas las personas ven lo mismo y de la misma manera.

Después de esta actividad se pasó a trabajar con el prejuicio altura, dividiendo al grupo de niños/as en dos subgrupos, elegidos por la altura. Un grupo estaba conformado por los alumnos de menor tamaño y el otro por los de mayor tamaño. Se llevaron carteles con dibujos de animales, una jirafa para el equipo de mayor altura y un mono para el equipo de menor altura. Se hicieron juegos breves para participar en equipo, por ejemplo, ir de un lado al otro caminando como mono, o ir de un lado al otro en puntas de pie. En los mismos había ocasiones en las que ganaron un equipo y otras el otro; por lo tanto, de esa manera se reflexionó que, a pesar de la diferencia de altura, todos pudieron jugar y divertirse, aceptando que somos distintos.

En el segundo taller se trabajó con el prejuicio peso; para esto se separó al grupo en 3 equipos y al azar se eligió a un integrante de cada grupo para que sus compañeros lo disfrazaran para parecer de mayor peso. De esta manera, los niños pudieron pensar que, a pesar de que sus compañeros cambien ficticiamente 
dentro del juego, o en la realidad, igual continúan siendo los mismos niños/as.

También se realizó el juego referido al prejuicio color de piel, a través de la realización de máscaras de material papel maché, realizadas la mitad de las mismas en color marrón y la otra mitad rosa. Éstas fueron entregadas aleatoriamente a cada niño y, al finalizar la dinámica, se las pudieron llevar. Se separó a los alumnos en dos grupos, diferenciados por color de máscara y se los hizo competir jugando, por ejemplo, con el ula-ula, o ir de un lado al otro saltando en un pie. Esto dio como resultado que en algunas ocasiones ganara un equipo y en otras el otro. Así, se logró reflexionar que, a pesar del color de piel que tenga cada uno, todos pudieron jugar y así debe ser también en la realidad, en la cual todos debemos divertirnos sin necesidad de discriminarse.

En el tercer taller se realizó el cierre de la intervención a través de la lectura de un cuento ilustrado, donde se relataba una historia con personajes de animales que querían organizar un partido de fútbol, pero al ser todos diferentes se generaban conflictos, los cuales luego pudieron resolverse satisfactoriamente para así poder jugar todos juntos (ver Anexos "Cuento: La Copa de la Selva"); de esta manera, se trataron temáticas relacionadas con los prejuicios y estereotipos de belleza. Luego de la lectura, con ayuda de los dibujos de los emoticones previamente utilizados, se realizaron preguntas para evaluar la comprensión del cuento y poder reflexionar sobre el mismo.

Al finalizar la realización de los talleres en el grupo experimental, se vuelve a tomar en ambos grupos la Escala de evaluación de prejuicios en la infancia, para observar si después del desarrollo de los mismos se dieron resultados positivos en estos niños, es decir, analizar si los prejuicios disminuyeron notablemente o no.

Por último, al obtener los resultados esperados, se realizó, por razones éticas y para que los demás pequeños tengan también la oportunidad de aprender, los talleres en el otro grupo.

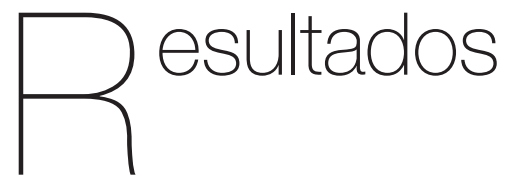

\section{Resultados del primer objetivo}

Como primer objetivo se propuso indagar, a través del estudio de las variables peso, altura, uso de anteojos y color de piel, los posibles prejuicios que pueden tener los niños.

Confeccionada la versión definitiva del instrumento Escala de evaluación de prejuicios en la infancia, se aplicó a dos salas de 5 años de pre-escolar, de una escuela pública de Godoy Cruz. Participaron un total de 42 niños, entre ambas salas. Aquella sala donde, a partir de las respuestas obtenidas a través del instrumento, se detectó mayor cantidad de elecciones basadas en prejuicios (sala Naranja), fue seleccionada como grupo experimental donde se aplicó la intervención (ver Figura 1).

Se administraba individualmente una tarjeta seguida de una pregunta, ante la cual el niño/a debía responder eligiendo una opción (A-B), una de ellas se 
encontraba referida al prejuicio a evaluar. Se aclara que la figura que representa una elección por prejuicio fue rotando en las sucesivas figuras. Las preguntas eran diferentes para cada figura, pero siempre aludían a realizar una elección según preferencia de juego o compañía.

En ambos grupos la cantidad de elecciones en base al prejuicio referido al sobrepeso fue un promedio de 84.5\%; con respecto al color de piel $72.5 \%$; altura 63.5\%; y, por último, en la variable uso de anteojos el promedio fue de $65.5 \%$. Por lo tanto, es importante destacar que la muestra sí demostraría tener prejuicios. Observándose con mayor cantidad de elecciones el sobrepeso, luego prejuicios debido al color de piel, uso de anteojos y altura en último lugar (ver Figura 1).

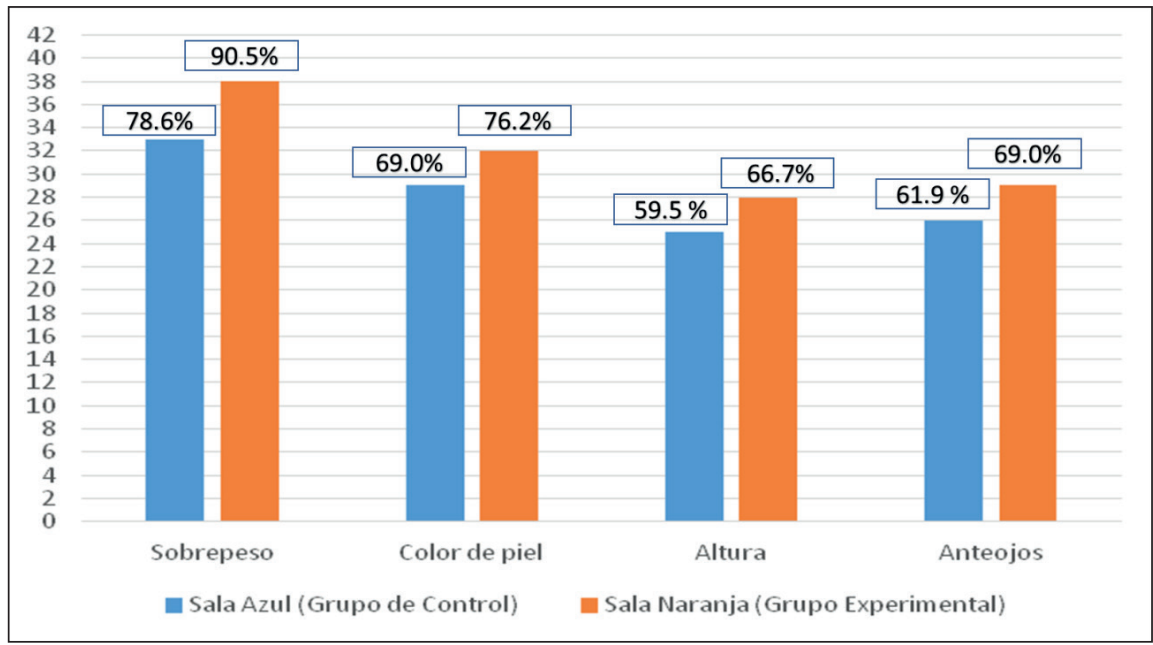

Figura 1. Comparación de elecciones pre-test en el Grupo Control y Experimental $(n=42)$.

\section{Resultados del segundo objetivo}

Como segundo objetivo se propuso comparar, a través de la realización de talleres, si los prejuicios estéticos cambian con respecto a su visión anterior. Se aplicaron talleres de intervención focalizados sobre los prejuicios evaluados. Se expone a continuación la frecuencia de elección basada en prejuicio en ambos grupos: experimental y control (ver Figura 2).

Como resultado, en el grupo experimental se observó que la elección basada en prejuicios disminuyó. En orden decreciente aquellas que más disminuyeron del pre-test al post-test fueron: discriminación por peso, color de piel, uso de anteojos, estatura. Mientras que en el grupo control, la elección por prejuicio aumentó en todos los casos, es decir, en todas las figuras.

Por lo tanto, los resultados obtenidos en ambos grupos muestran que los talleres de intervención resultaron de utilidad, ya que, a partir de éstos, los niños demostraron una disminución en los prejuicios a evaluar. 


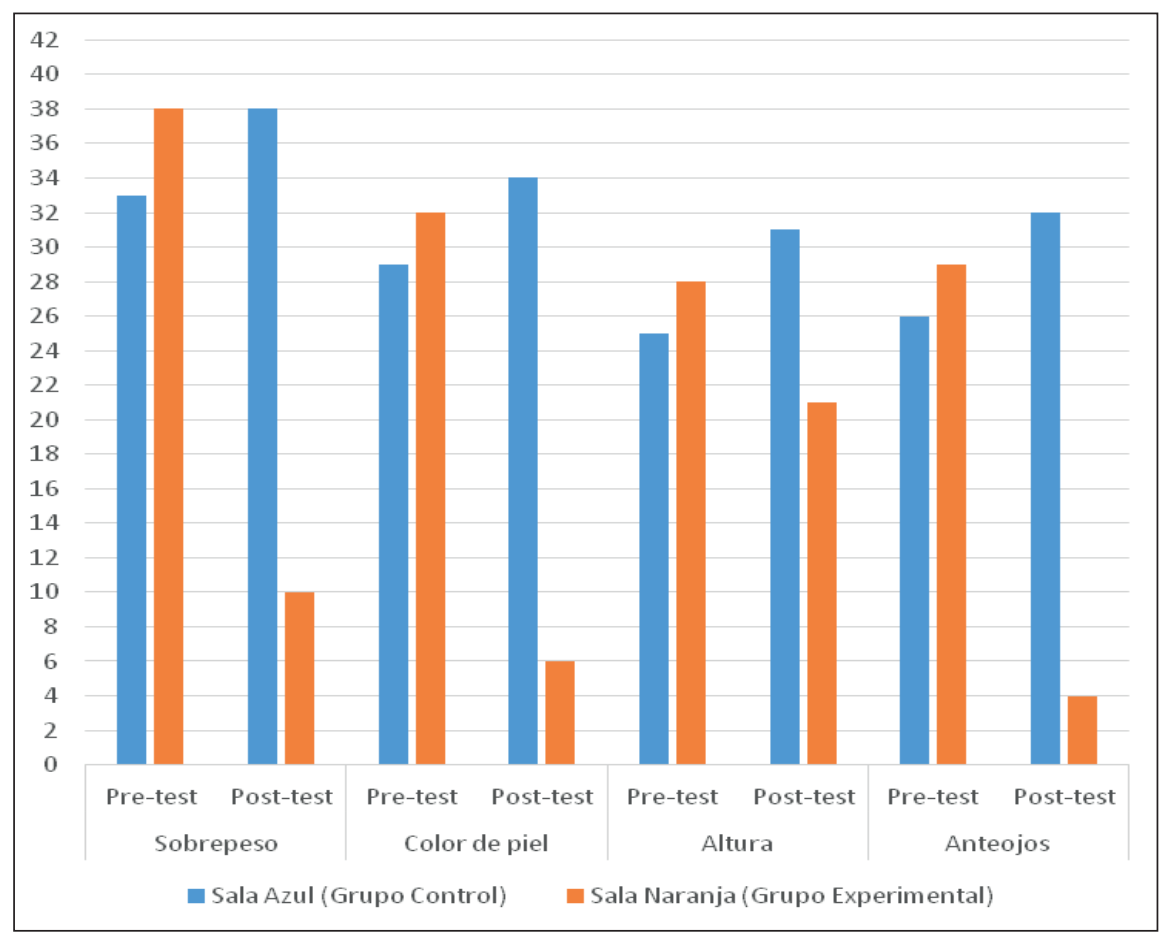

Figura 2. Comparación de elecciones pre - post en el grupo Control y Experimental $(n=42)$.

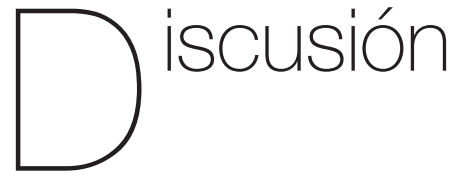

En el primer objetivo específico se propuso indagar a través del estudio de las variables peso, altura, uso de anteojos y color de piel, los posibles prejuicios que pueden tener los niños/as.

A partir de la administración del instrumento, se observa como resultado que la cantidad de elecciones basadas en prejuicio, entre los dos grupos (control y experimental) fueron: sobrepeso $84.5 \%$; color de piel $72.5 \%$; altura $63.5 \%$ y uso de anteojos 65.5\%. Por lo tanto, puede destacarse que los estudiantes demostrarían tener prejuicios; observándose con mayor cantidad de elecciones el sobrepeso, luego por color de piel, uso de anteojos y altura en último lugar.

Entre las edades de tres y seis años, los niños comienzan a entender y utilizar los estereotipos (Elizathe et al., 2011; Lobera et al., 2008; Pappas, 2012). Después de los seis años, comprenden los estereotipos de otras personas; más exactamente antes de los cinco años, la inmensa mayoría de niños y niñas expresan actitudes polarizadas hacia la complexión física y, a partir de esa edad, crecen rápidamente los estereotipos negativos, expresándose de forma abierta y sin matices (Abrams, 2010; Raabe \& Beelmann, 2011; Solbes et al., 2008).

El prejuicio que resultó con mayor cantidad de elecciones fue el relacionado con el peso; es importante mencionar que la existencia de actitudes negativas hacia el 
sobrepeso se demuestra en dos grupos de niños en edad preescolar de tres a cinco años. Los estudios muestran que los prejuicios hacia el exceso de peso han ido aumentando en la sociedad occidental, tanto en adultos como en niños, y que éstos internalizan muy pronto las normas e ideales de belleza asociados a la delgadez (Cramer et al., 1998; Solbes et al., 2008).

Y como el segundo prejuicio en orden de elección fue el relacionado con el color de piel, se destaca que, ya en primer grado de la etapa escolar, los niños comprenden que la discriminación basada en raza o color de piel, es moralmente incorrecta. Por lo tanto, comenzar a trabajar en ella desde ese momento sería lo recomendado (Brownell et al., 2005; Lobera et al., 2008; Pappas, 2012).

Dentro del segundo objetivo se propuso comparar, a través de la realización de talleres, si los prejuicios estéticos cambian con respecto a su visión anterior.

Como resultado, en el grupo experimental los niños optaron en mayor medida por elecciones sin base a prejuicios, a diferencia de lo ocurrido con los niños del grupo control donde la tendencia fue inversa: las elecciones con base a prejuicios aumentaron.

La discriminación debido a aspectos físicos se define como la creación y/o difusión de estereotipos por características reales o imaginarias, sean éstas positivas o negativas y que se vinculen con particularidades físicas innatas o adquiridas. Ésta se caracteriza por poseer un solo modelo de belleza que resulta opresivo, de burla y discriminación a quienes no se ajustan a ese modelo arbitrario (Elizathe et al., 2011; INADI, 2011).
A pesar de que todos seamos diferentes, todos tenemos derechos iguales. Cualquier aspecto puede servir como excusa para molestar a otra persona o burlarse de ella. Puede ser porque no usen ropa de moda, por su color de piel, quizás porque estén pasados de peso o usen anteojos. Esto se denomina discriminación y los niños o niñas que molestan o amenazan a otros/as, no necesitan mucho para inspirarse si tienen la intención de herir o dejar a alguien fuera de su círculo de amigos (Elizathe et al., 2011; INADI, 2011).

Esta investigación se decidió llevar a cabo dentro del ámbito escolar, ya que el proceso educativo no debe encaminarse exclusivamente a la adquisición de conocimientos ni al entrenamiento del razonamiento formal, sino también y, sobre todo, a la enseñanza de valores. Reflexionar sobre los prejuicios dentro del proceso de educación a través de la enseñanza de esta temática no es solamente hablar sobre ella sino hacerla (practicarla). De esta práctica surgirá la reflexión o el pensar sobre los prejuicios. Es importante adelantarse a los hechos, por eso, esta enseñanza se debe implementar a través de programas de prevención del acoso escolar que se inicien en los primeros años de la escolaridad, en nivel inicial; esperar hasta los 6 años, o la primaria, es demasiado tarde, y que éstos se continúen hasta el último año, promoviendo un trabajo continuo. Igual de importante es la educación desde la familia, ya que constituye la matriz psicosocial donde el niño comienza y continúa su proceso de desarrollo (Abrams, 2010; Lebria et al., 2016).

Se debe reconocer como limitación de esta investigación el hecho de no haber podido evaluar la estabilidad de los cambios logrados, es decir, si los niños y niñas 
mantuvieron las elecciones a lo largo del tiempo, al menos 3 meses, después de la intervención.

Como sugerencia, principalmente se debe mencionar la importancia de realizar dicha educación en prejuicios y valores durante todo el ciclo lectivo, para que los niños lo incorporen como una currícula anual y puedan internalizar los conceptos e ideas desde el inicio de la infancia.

También para futuras intervenciones se destaca que estos talleres podrían llevarse a cabo en una mayor cantidad de establecimientos educativos de diversos contextos socio-culturales, para, de esa manera, aumentar el número de participantes y, así, lograr generalizar la temática para generar un posible cambio a nivel social.

Comunicar y educar en la diversidad nos invita a reflexionar y distinguir que lo opuesto a la igualdad no es la diferencia, sino la desigualdad. De la misma manera, equivocadamente se usa la palabra "tolerancia", como antidiscriminatoria. Tolerar, etimológicamente significa soportar, por lo que "tolerar" e "incluir" están muy lejos de ser sinónimos; mientras la inclusión reconoce las diferencias y busca la igualdad de trato y oportunidades, la tolerancia aparece como el lugar de quien tiene una jerarquía superior, una mirada sesgada del "normal" hacia el "diferente". La diferencia no debe ser tolerada sino respetada (INADI, 2011).

En conclusión, a partir de los resultados obtenidos se pudo observar que se lograron disminuir los prejuicios en los niños y niñas. Por lo tanto, es bueno tener en cuenta este aspecto favorable de la investigación, ya que, de esta manera, los niños quizás pudieron aprender e interiorizar una perspectiva distinta a la que poseían de observar a las personas, de una manera que no sea prejuiciosa ni dañina para el otro, sino a través de una visión ampliada y sin estereotipos perjudiciales de por medio. Para así, de a poco, lograr que los niños puedan ser educadores de sus propios compañeros y padres.

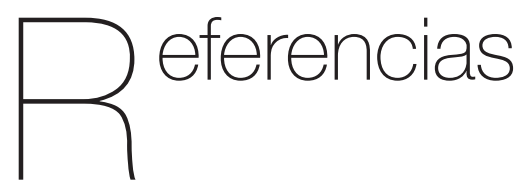

Abrams, D. (2010). Processes of prejudice: Theory, evidence and intervention. Manchester: Centre for the Study of Group Processes, University of Kent.

Brownell, K., Puh, R., Schwartz, M., \& Rudd, L. (2005). Weight Bias: Nature, Consequences and Remedies. (1 $1^{\mathrm{a}}$ ed). New York: Guilford Press.

Clark, K., \& Clark, M. (1947). Racial identification and preference in Negro children. In T. M. Newcomb \& E. L. Harley (Eds.), Reading in social psychology (pp. 169-178). New York: Holt Rinehart and Winston.

Cramer, P., Steinwert, T., \& College, W. (1998). Thin is good, fat is bad. How early does it begin?. Journal of Applied Developmental Psychology, 19(3), 429-451.

Doyle, A. B., Beaudet, J., \& Aboud, F. E. (1988). Developmental patterns in the flexibility of children's ethnic attitudes. Journal of Cross-Cultural Psychology, 19, 3-18.

Elizathe, L., Murawski, B., \& Rutsztein, G. (2011). La Cultura de la delgadez en los 
niños. Discriminación y estigmatización social. Revista Encrucijadas-UBA, 50(3), $1-6$.

Guerrero, S. (2007). El desarrollo de la toma de conciencia racial. Un estudio evolutivo con niños españoles de 3 a 5 años. (Disertación doctoral no publicada), Universidad de Castilla- La Mancha Facultad de Psicología, Departamento De Psicología Evolutiva y de la Educación, Cuenca, España.

Hernández-Sampieri, R., Fernández-Collado, C. \& Baptista-Lucio, P. (2014). Metodología de la Investigación. (6ta Ed.). México D.F.: Mc Graw Hill.

Horowitz, E. L. (1936). The development of attitude toward the negro. Archives of Psychology, 194, 8-36

Instituto Nacional contra la Discriminación, la Xenofobia y el Racismo [INADI]. (2011). Somos iguales y diferentes. Guía Didáctica para Docentes Guía para niñas y niños de prevención de prácticas discriminatorias. Buenos Aires: Argentina.

Katz, P. A., \& Zalk, S. R. (1978). Modification of children's racial attitudes. Developmental Psychology, 14, 447-461

Lebria, C., Alamilla, E., León, R. \& Hermosillo, A. (2016). El papel de la familia y de la escuela en la formación de valores. Revista Electrónica de Psicología Iztacala, 19(4), 1355-1380.

Lobera, I., Rivas, M., Montaña, M., \& Morales, M. (2008). Influencia de los estereotipos en la percepción de la obesidad. Nutrición Hospitalaria, 23(4), 319-325.
MentalHealth.net (2010). Prejudice. Recuperado de https://www.mentalhelp. net/articles/prejudice/

Montero, I., \& León, O. G. (2007). A guide for naming research studies in Psychology. International Journal of Clinical and Health Psychology, 7(3), 847-862.

Pappas, S. (2012). Young children follow adults' leads on prejudice and stereotypes. New York: Live Science Contributor.

Raabe T. \& Beelmann, A. (2011) Development of ethnic, racial, and national prejudice in childhood and adolescence. A multinational meta-analysis of age differences. Child Development, 82(6), 1715-1737. doi: 10.1111/j.1467-8624.2011.01668.x.

Richardson, S. A., Goodman, N., Hastorf, A. H. \& Dornbusch, S. M. (1961). Cultural uniformity in reaction to physical disabilities. American Sociological Review, 26, 241-247

Solbes, I. (2010). El sobrepeso en la niñez y sus consecuencias sociales. Un estudio evolutivo sobre las actitudes explícitas e implícitas hacia la complexión física y su relación con la imagen corporal. (Tesis de Doctorado). Facultad de Psicología Universidad Complutense de Madrid, Madrid, España.

Solbes, I., Enesco, I., \& Escudero, A. (2008). Los prejuicios y las actitudes negativas hacia la gordura en la infancia. INFAD Revista de Psicología, 1, 251-260.

Williams, J. E., Best, D. L., Boswell, D. A., Mattson, L. A., \& Graves, D. J. (1975). Preschool Racial Attitude Measure II. Educational and Psychological Measurement, 35, 3-18. 